\title{
La visualización femenina en la pesca artesanal: transformaciones culturales en el sur de Chile
}

\author{
María Catalina Álvarez
}

Universidad de Los Lagos, Osorno, Chile. Email: catalina.alvarez@ulagos.cl

\section{Galicia Stuardo Ruiz}

Universidad de Los Lagos, Osorno, Chile. Email: galiana23@gmail.com

\section{Daniela Collao Navia}

Universidad de Los Lagos, Osorno, Chile.

Email: danielacollaonavia@gmail.com

\section{Claudio Gajardo Cortes}

Universidad de Los Lagos, Osorno, Chile.

Email: clgajardocortes@gmail.com

Resumen $^{\mathbf{1}}$ :La mujer en la pesca artesanal ha desarrollado su quehacer desde una cotidianidad paralela a los grandes modelos de desarrollo, siendo invisibles su quehacer frente a las tensiones y crisis ambientales y productivas. Esta investigación tiene como objetivo el describir la participación de la mujer en la pesca artesanal chilena, utilizando revisión de fuentes secundarias, revisión bibliográfica y notas etnográficas. Analíticamente, estos antecedentes son leídos desde dos enfoques; i) desde las transformaciones productivas y territoriales, y ii) entendiendo a la pesca artesanal como un sistema cultural. La descripción destaca los roles de la mujer en los procesos de transformación, así como sus saberes y prácticas ambientales, en futuros escenarios que las reconozca como una alternativa critica que desde los espacios domésticos que trascienden a las lógicas administrativas de extracción. turales.

Palabras clave: Visualización de la mujer, pesca artesanal, sistemas cul-

\section{Women display on artisanal fisheries: cultural tranformation ont he south of Chile}

Abstract: When it comes to artisanal fishing, traditional development models dealing with environmental and productive crisis do not take into account the perspectives of women and their everyday life. The objective of this research is to 
describe the participation of women in Chilean artisanal fishing sector, using reviews from secondary sources, bibliography reviews and ethnographic notes. Analytically, these records are reads from two points of view; i) from productive and territorial transformations, and ii) understanding artisanal fishery as a cultural system. The description highlights the role of women in the transformation processes, as well as their knowledge and environmental practices, in future scenarios that recognize them as a critical alternative that transcends the administrative logics of extraction from domestic spaces.

Keywords: Womandisplay, artisanalfishieries, cultural systems.

\section{Visualizando as mulheres na pesca artesanal: transformaçõesculturais no sul do Chile}

Resumo: As mulheres na pesca artesanal tem desenvolvido o seu trabalho a partir de um quotidiano paralelo aos grandes modelos de desenvolvimento, sendo invisível seu trabalho perante as tensões e crises ambientais e produtivas. Esta pesquisa tem como objetivo descrever a participação das mulheres na pesca artesanal chilena, usamdo a revisão de fontes secundárias, revisão de literatura e notas etnográficas. Analiticamente, esses registros são lidos a partir de duas abordagens; i) a partir das transformações produtivas e territoriais, e ii) entendendo a pesca artesanal como um sistema cultural. A descrição salienta o papel das mulheres nos processos de transformação, assim como seus conhecimentos e práticas em cenários futuros em que sejam reconhecidas como uma alternativa crítica que desde os espaços domésticos transcendam às lógicas administrativas de extração.

Palavras-chave: Visualizaçãoda mulher, pesca artesanal, sistemas culturais.

\section{Introducción}

Los territorios costeros, son una construcción cultural, reflejo de la constante interacción entre comunidades indígenas, pescadores artesanales, agricultores, intermediarios, recolectores y acuicultores, dentro de los cuales las mujeres han sido co-protagonistas en la pesca artesanal, viviendo desde los espacios domésticos y administración, comercialización (Frangoudes\&Keromnes, 2008; Rubio-Ardanaz, 2005), recolección, elaboración de artes de pesca y seguridad alimentaria (Ram-Bidesi, 2015). No obstante, consecuencia de las transformaciones contemporáneas, es que los roles de las mujeres han tendido a hacerse visibles, como un actor con alternativas al desarrollo de la pesca artesanal.

Las transformaciones contemporáneas en la pesca artesanal chilena son a causa de las presiones del mercado y las regulaciones de la actividad. Sus consecuencias son transversales y han sido registradas en migraciones interregiones, o entre sectores productivos, así como la disminución en la biodiversidad marina producto de la sobre explotación y contaminación.

En este escenario, los roles de las mujeres en la pesca artesanal son observados desde dos enfoques; i) la pesca artesanal como un sistema 
económico-productivo y ii) la pesca artesanal como sistema cultural. Estos enfoques nos permiten dar cuenta de una caracterización de sus prácticas y llevarlas a una reflexión que permitan imaginar escenarios de futuro en instancias de intermediación en políticas públicas.

\section{Enfoque económico-productivo}

\section{Transformaciones en los territorios costeros}

Hacer visible la participación de las mujeres en la pesca artesanal en las transformaciones territoriales costeras, implica reconocer su trayectoria, permitiendo comprender sus potencialidades y proyección de alternativas al desarrollo y la sustentabilidad. Cada territorio costero y los/as pescadores/as que lo habitan son heterogéneos, por ende las transformaciones a pesar de que pueden ser observados como transversales, no es posible hacer generalizaciones de las respuestas locales que ahí ocurren (Woortmann, 2007). En Chile es posible reconocer tres procesos transversales en los territorios costeros: i) Transformaciones normativas, ii) transformaciones en actividades productivas, iii) transformaciones del mercado.

Las i) transformaciones normativas,desde la década de los noventa a la fechahan significado un crecimiento en la regulación de las prácticas en las actividades pesquero artesanales, regulando las actividades categorizadas como legales y otras como ilegales. La mujer ha debido adaptar sus prácticas cotidianas entre ambos mundos. Un ejemplo de esto es lo que ocurre con la pesca de descarte. Las legislación regula qué especies están autorizadas para ser extraídas, su tamaño y su cantidad, no obstante, la fauna acompañante tiene una menor regulación (Subpesca, 2015), y muchas de ellas no se encuentran autorizadas para su comercialización, como ejemplo la pitufa, merluzas de menor tamaño que no pueden ser declaradas y son aprovechadas para la cocina familiar, intercambio, o congelados para el comercio local a menor escala.

Sobre las ii) transformaciones en actividades productivaslas mujeres se han caracterizado por desenvolverse en espacios consolidados históricamente, como la producción local de pequeña agricultura, recolección de algas y mariscos (Belkis, 2012), elaboración y reparación de artes de pesca, e intercambio y comercialización local, entre otros (Harper et al., 2013). Estas actividades normalmente no son asalariadas, no obstante en las últimas décadas han disminuido y reemplazadas por labores asalariadas industriales, donde la mujer tiene poder adquisitivo que antes no, lo que provoca tensiones sobre los roles (Rebolledo, 2012; Yáñez, 2010). La actividad de recolección de alga se ha visto limitada por la escases de biodiversidad, creciente contaminación y concesiones acuícolas (Andrade, Arenas y Guijón, 2008). Algunas de los ejemplos gráficos de esta transformaciones es el abandono de los campos y el mar que hombre y mujeres han hecho para enfocar sus esfuerzos laborales en industrias que otorgan un sueldo estable y claro. Algunas de esas industrias son desconchados y 
salmoneras, quienes en su mayoría contratan a mujeres para trabajos de limpieza en la cadena de procesamiento de los recursos (Torres y Saavedra, 2014; Saavedra, 2015).

Finalmente, una transformación fundamental ha sido la iii) apertura al mercado del Estado, que consiste en una transformación en el sistema económico nacional, en la que la producción y demandan se encuentran reguladas por las demandas nacionales y sobre todo internacionales, la que se caracteriza por una baja participación del Estado como agente regulador (Hidalgo, Ther y Saavedra, 2013). Consecuencias de esto, la vida cotidiana de la mujer en territorios costeros han requerido de su incorporación a los espacios públicos, incluyendo innovación en las estrategias de adaptación por medio de emprendimientos empresariales, o bien como procesos regionales (unidad político administrativa) en relación a las actividades productivas frente a las respuestas del mercado (McPhee, 2013). Algunas de las estrategias de adaptación han sido las modificaciones de casas en hospedajes para trabajadores de las industrias salmoneras, o la migración de la mano de obre hasta regiones extremas, como Provenir en la Región de Aysén y Magallanes de mujeres desde la región de la Araucanía, para el trabajo en plantas procesadoras² (Arteche, 2014; Chávez y Milihuichún, 2011).

\section{La división sexual del trabajo como base de la desigualdad en la pesca artesanal}

La historia de la pesca artesanal en Chile ha sido concebida como actividad marcadamente masculina, donde la participación de las mujeres es abiertamente como un actor secundario, invisible (Gordon, 2014).

Hirata y Kergoat (2007), exponen que la división de labores experimentada históricamente desde la pesca artesanal, tiene su base en un sistema de división sexual del trabajo que sitúa a hombres en el espacio productivo y a mujeres en el reproductivo, que marca una escala jerárquica con agrega valor a las actividades productivas. La concepción de la mujer como “actor secundario” debe ser revisada, debido a su importante inserción en el mercado de trabajo de proceso y actividades asociadas. Las áreas de desarrollo productivo en la pesca artesanal, que se caracterizan por una marcada inserción de mano de obra femenina son; i) la recolección orilla, ii) trabajo en plantas de proceso y iii) actividades asociadas, de valor agregado o turismo.

La actividad de recolección de orilla ha sido en el transcurso de la historia de la pesca artesanal, una actividad en la que se han insertado en mayor medida la mujeres, ya sea para la venta de productos, como para el consumo del hogar. Sin embargo, este tipo de extracción, si bien ha tenido momentos altos de productividad, en la actualidad se encuentra en una baja considerable, que es resultado, entre otras cosas, de importantes mermas en la cantidad de recursos y de transformaciones en los mercados asociados (Guerra y Skewes, 2010). De esta forma se configura como una activi- 
dad de bajos ingresos y condiciones inestables de productividad, sumándose a esto la desprotección a niveles de seguridad y salud que tienen este tipo de trabajos particulares. No obstante, los datos entregados por Subpesca, del total de mujeres inscritas en el Registro Pesquero Artesanal (RPA), el 87,8\% de estas se dedica a la recolección, particularmente de algas (Subpesca, 2014), y que hoy en día se han volcado a la acuicultura en pequeña escala, con el afán de asegurar la productividad del medio y así sólo el 6\% de titulares de concesiones acuícolas son mujeres (Subpesca, 2014).

El trabajo en Plantas de Proceso, por otra parte, se configura como parte del proceso de modernización de esta misma (Arteche y Saavedra, 2014). En esta rama, del total de 10.007 trabajadores, 3.780 o sea el $37,8 \%$ son mujeres, mientras que 6.219 son hombres, esto es el 62,2\%. Sin embargo, en relación al régimen laboral de mano de obra permanente los hombres representan el $71 \%$ del subtotal, mientras que en el trabajo casual, las mujeres son mayoría, con un 54,8\% de su subtotal (Subpesca, 2014). De esta forma, se observa que si bien su participación es relevante, estas siguen ocupando puestos de trabajo que presentan mayores condiciones de vulnerabilidad laboral.

En relación al trabajo en actividades asociadas a la pesca, como lo son el turismo, se observa a nivel nacional que las mujeres ocupan un rol fundamental, cumpliendo labores principalmente en áreas administrativas y gastronómicas (Pilquiman y Skewes, 2010; Pilquiman y Skewes, 2009). El turismo y actividades asociadas a la pesca artesanal, en conjunto con la acuicultura, se han convertido, en los territorios costeros, en una de las alternativas productivas más recurridas para sobrellevar la crisis económica producida por los escases de recursos; y son además las nuevas áreas que están siendo impulsada por los aparatos del Estado, para apalear los efectos de esta crisis. Debido a esto, muchas de las economías locales se han volcado al desarrollo de emprendimientos turísticos que complementan o, en algunos casos, reemplazan a la actividad extractiva (Guerra et al, 2010). Bajo estas condiciones, la mujer se convierte en una de las principales proveedoras del hogar, rompiendo con las lógicas de género establecidas en la sociedad occidental.

Finalmente, los datos que entrega Subpesca (2014), de 20.401 mujeres inscritas en el RPA, solo 1.641 tienen participación en las organizaciones sindicales o gremiales identificadas, equivalente al $8 \%$ de participación,mientras que del total de participación en organizaciones sindicales o gremiales, entre hombres y mujeres, las mujeres representan apenas el $12 \%$ del total. El porcentaje de participación de mujeres en organizaciones, responde también a que la presencia de estas en el rubro es mucho menor que la de los hombres (Cid, 2012). Sin embargo esta situación nos abre un problema, y es que dentro de los registros nacionales sobre pesca artesanal, las áreas complementarias o de procesamiento en las que se desenvuelven las mujeres, no son consideradas como parte de la actividad. Una de las consecuencias más importantes de este hecho, es que la mayor 
parte de las mujeres que participan de la pesca artesanal dentro de las actividades complementarias, quedan fuera de las esferas de organización $\mathrm{y}$, por lo tanto, de la toma de decisiones con respecto a la actividad pesquera.

Sin embargo, desde las últimas dos décadas, federaciones de pescadores artesanales, instituciones de estado, fundaciones y ONG's afines, han impulsado una serie de encuentros latinoamericanos y nacionales, que buscan reflexionar sobre el rol de las mujeres en la pesca artesanal y dar solución a algunas de las problemáticas identificadas desde estos espacios. Estos encuentros han girado en torno al reconocimiento de la crisis pesquera artesanal y la necesidad de avanzar hacia la sustentabilidad de esta misma, considerando esta actividad productiva como uno de las claves para asegurar la alimentación de las poblaciones mundiales (Colectivo Internacional de Apoyo a los Pescadores Artesanales, 2008). En este contexto, se apuesta a reconocer el rol relevante que cumplen las mujeres en aportar a nuevas formas de sustentabilidad mediante su trabajo y conocimientos tradicionales. Se reconoce y reflexiona además, sobre las condiciones de vulnerabilidad en las que se encuentran laboralmente, donde los bajos sueldos y condiciones laborales precarias, se suman a las responsabilidades que estas asumen con el trabajo doméstico; labor que debido a la división sexual del trabajo imperante, se mantienen como actividad exclusiva de mujeres.

Por tanto, las labores que desarrollan las mujeres en el mundo laboral relacionado a la pesca artesanal, responden a ciertos estereotipos que encasillan las capacidades y roles de estas en la esfera privada/doméstica, trabajos relacionados al procesamiento de recursos y administración, que se asocian al mantenimiento y administración del hogar. Esta división ideológica de labores basadas en el sexo, han contribuido a la reproducción de las condiciones de desigualdad entre hombres y mujeres, a pesar de la relevancia que han ido ocupando estas últimas en las economías locales, dejando en una posición de inferioridad a las mujeres (Hirata y Kergoat, 2007; Abramo, 2004).

\section{Enfoque cultural}

\section{Saberes y prácticas de mujeres en la pesca artesanal}

El conocimiento sociocultural, resulta pertinente para comprender la presencia de la mujer en diversos ámbitos, siendo capaz de visibilizar sus prácticas desde un lugar más abierto que sólo los criterios del desarrollo económico.

$\mathrm{Al}$ respecto, la crisis de la pesca artesanal ha sido caracterizada tanto por la escasez de recursos marítimo-costeros, la contaminación y tecnologización del mar, pero también por la falta de políticas y perspectivas que puedan comprender y actuar desde las propias dinámicas socioculturales, que sean capaces de sumar la economía familiar pesquera y 
formas de habitar de la mujer, como transversal a la vida en las sociedades litorales (Breton y Doyon, 1999). En otras palabras, se trata de evidenciar prácticas que nos permitan una concepción más amplia para encontrar nuevos itinerarios.

Para otorgar un sentido abierto a las relaciones entre el territorio, como construcción cultural y el medio ambiente, es fundamental la descripción etnográfica de las prácticas de mujeres en la pesca artesanal, más allá de la microempresa como única forma de empoderamiento o valoración (Rodríguez, 2011; López, 2011; García, 2001; Maneschy; Siquira y Miranda, 2012).

Desde el punto de vista técnico el trabajo de pescador requiere conocimientos específicos que son comunes tanto a hombres como a mujeres en las mismas prácticas, pero además hay una gama de saberes sustentados específicamente por mujeres. A continuación se destaca una mirada a prácticas, aprendizajes y conocimientos, en la vida social en las comunidades, donde las mujeres se insertan en la vida social de las Caletas de pescadores, aportando nuevos temas a los procesos de manejo y uso de los recursos naturales alternativos al modelo productivista y de desarrollo.

El primer caso es el de Margarita, habitante de Caleta El Manzano, comuna de Hualaihué en la zona de Chiloé continental. Normalmente, Margarita vende carne de ovejas a sus vecinos y para criar estos animales ella los deja en una zona de la playa que se encuentra aledaño a su casa y donde crece una especie de pasto costero que llaman Llipé. Éste sector, además es utilizado para la crianza de animales por los demás habitantes de la caleta siguiendo reglas específicas. El espacio no tiene propiedad específica y es considerado patrimonio común. Cuando alguien quiere dejar sus ovejas en el sector, se supone que debe ser parte del cuidado de todos los otros animales. Cuando las ovejas se desplazan mucho hacia la costa son arreadas antes que suba la marea. Desde decenas de años ha utilizado ésta zona y es de las más antiguas en ello. De hecho, su hermana y sus cuatro hermanos, cada uno de ellos en hogares diferentes, también utilizan este sector desde siempre de modo que son ellos en quienes se concentran las prácticas de uso común. La diferencia ha sido que los hijos de los hermanos hombres no han seguido la tradición, mientras que los hijos de ambas mujeres poseen animales y participan de las prácticas comunes. Así, éste sector de pastoreo se torna de uso común al entenderlo como compuesto de una estructura de relaciones de reciprocidad que a nivel de la caleta es más potente entre los hermanos, la que también se difunde hacia los otros habitantes pero donde Margarita y su hermana están siendo quienes dan continuidad y reproducción a éstas prácticas en las generaciones futuras.

El segundo caso es el de Miryam, habitante del borde costero noreste de la isla de Chiloé, quien aprendió la recolección de algas y mariscos observando cuando su padre hacía recolección por la costa. En Guabún, las casas están en colinas frente al mar. Para ir a recolectar, debe subir y bajar por la colina por un sendero media hora. Recordar si la marea está subiendo 
o bajando le ayuda para saber si dejar el almuerzo y otros tiempos organizados en la noche anterior, así mientras deja las algas secando puede volver a almorzar rápidamente y volver a recoger lo que se secó. Antes de salir, evalúa el viento y escucha el sonido del mar desde su casa, porque si la marea está pegando de frente no es conveniente recolectar. El sendero lo usan los otros miembros de su familia. Y en la Caleta hay otros cuatro o cinco senderos ubicados en otras colinas utilizados por otras algueras. Si se encuentran en la playa, pueden intercambiarse pasando entre playa y playa por la costa porque cuando ya se realizó el corte en una, se informan o se observan para que no se repitan. Con algunas algas como el Llapín, saben incluso en cuánto tiempo se renuevan y allí vuelven. La repartición de acceso familiar a las playas, está sustentado sobre la base de relaciones de algueras. Nuevamente, es un uso consensuado del espacio y sus recursos que sirve como una defensa frente a pescadores de otros sectores y sobreexplotan el lugar. En definitiva, las algueras envuelven al espacio geográfico costero convirtiéndolo en una apropiación sociocultural de la que participa toda la sociedad litoral. Cuando alguien pide permiso para entrar por un sendero lo consigue; "porque si es para comer no se le niega el permiso”.

Un tercer caso lo encontramos en Lucy, igualmente habitante de la tranquilidad de la Caleta Guabún. "También estuve en Santiago una vez, era por quince días el paseo, y yo me vine nomas a los diez días”. Aquí se siembra papa cuando la luna está llena -me responde- pero nunca han sembrado con luna mala, asique no saben si es cierto. Sirve además para los Chanchos que comen Llapín con papa molida. Tiene 55 años aprox. Para vender mariscos en Ancud, con una aguja de madera de Luma enhebrada con pita de junquillo ensarta por la boca catorce Chaperines que forman un collar, y dos collares forman la sarta que vale 1.500 aprox. El cochayuyo que se come se corta más arriba de la raíz, con Ulte y todo, se lava y se cuece. El que se vende se corta más arriba y se pone en las piedras al sol, para que quede más claro, amarillo. Para el cangrejo sólo de consumo familiar, se usa una pita con Lapa de carnada, cuando está comiendo agarrado de la lapa se saca arriba. La Luga se vende harto pero el sargazo no ha dejado crecer mucha luga porque le da mucha sombra. Cuando no hay Luga se saca el Llapín que se deja crecer cada seis días. También saca Luche. El luche hay que secarlo y hacerlo panes. Se seca en el pastito o mallita para que no se ensucie, como es comestible tiene que tener un cuidado especial. No tiene que quedar muy seco, ni muy mojado. Después uno calcula tres puñados de luche y se envuelve en un papel de diario y se amarra con locha y se deja en el horno hasta que se cocine.

Los conocimientos y prácticas otorgan acceso al espacio y los recursos, que a su vez, pasan a ser concebidos y usados por toda la sociedad. Se trata de tramas de conocimientos no necesariamente originados sólo por hombres o mujeres, pero que sustentan lo particular de cada técnica y producto elaborado, que están implícitas en el lugar, y mantienen la vida social. La actividad extractiva y de procesamiento, conocidas por las instituciones de fomento productivo, son inferidas de una cotidianidad donde se resalta 
deliberadamente, en términos de desarrollo económico, actividades de recolección de mariscos en los roqueríos o su proceso en el hogar como alimento o para la venta, pero entre éstos quehaceres hay una cadena de prácticas, conocimientos y preocupaciones mucho más densa. Así, las prácticas y saberes de estos casos incluyen la elaboración de productos y la extracción, pero no se agotan exactamente en esto.

La percepción adquirida por la mujer se puede diferenciar por la planificación de futuro, pues allí donde los conocimientos han sido creativamente impresos en el ambiente un modo de hacer las cosas refleja cómo cada día se satisface necesidades ineludibles y previstas. Ésta visión se forma de habilidades adquiridas que van más allá de cierta asignación de rol, o que al menos lo sobrepasan. Es ésta visión la que no puede ser excluida de la innovación, porque se trata de un arraigo que pone a raya el carácter maximizador del mercado en tanto que atenta contra las condiciones del territorio, desde la reproducción familiar a todas las condiciones ambientales.

La asignación cultural de roles domésticos en la estructura social y la necesidad de comprender nuevas variaciones en las prácticas puede ocurrir al considerar el que sobre los propios quehaceres de la mujer han aparecido diversos usos y aprendizajes que derivan en otros temas. En el caso de sociedades vinculadas a ecosistemas costeros donde esto también es patente, la mujer logra una apropiación del ambiente diferenciada, lo defiende más que nada por las necesidades de mantener la vida de la familia y los hijos en el futuro. No se trata de que esto siga siendo así, sino de que ahora, esto corresponde a una visión centrada en la reproducción de la vida y un completo aporte a la sustentabilidad. Una apropiación de la naturaleza motivada por una percepción de futuro que articula los recursos naturales, el habitar y las necesidades fundamentales del hogar otorgando visiones de la economía pesquera que no agotan su aporte sólo en el emprendimiento y productividad, o la articulación de la mujer a los mercados dinámicos.

\section{Futuros escenarios en espacios públicos}

Los organismos internacionales e instituciones en Chile han incrementado su preocupación sobre la participación de la mujer en la pesca artesanal. La Unión Europea, financia esfuerzos que han permitido obtener un retrato relativamente contundente de la situación de las mujeres en la pesca artesanal en Europa. En 2001 publica el Libro Verde sobre el futuro de la política pesquera común (PPC). El texto admite la necesidad de contribuir al reconocimiento y fomento del importante papel que desempeñan las mujeres en él, solicitando incrementar las medidas que fortalezcan el papel femenino. En 2002, se establece una serie de recomendaciones específicas respecto a la formación y apoyo a mujeres al incorporar un valor añadido a su producción. En el año 2003 patrocinó una conferencia sobre "El lugar de las mujeres en el sector de la pesca”, destacando los obstáculos que impiden su progreso en estructuras inadecuadas, además de los diversos roles en que participan, publicado en el informe "Pesca europea las mujeres en la 
pesca: un papel desconocido" (Colectivo Internacional de Apoyo a los Pescadores Artesanales, 2008), entre los que destacan parte de la tripulación, plantas de transformación, proveedores, investigadores, hasta las responsables de la transmisión de conocimiento, es un reconocimiento a su participación como una muestra cultural de la apropiación de la actividad pesquero artesanal y no solo una forma de empleo”. Ese mismo año se presenta una convocatoria de propuestas destinada específicamente a las mujeres del sector pesquero, cuyo objetivo fue fomentar la creación de redes, el intercambio de experiencias y el desarrollo de actividades de formación.

En Chile mientras tanto, en 2007 el gobierno invita al Primer Encuentro Internacional de Mujeres de la Pesca Artesanal y Acuicultura de Pequeña Escala, organizado por el Fondo de Fomento de la Pesca. El objetivo del encuentro fue el reconocimiento de la existencia de la mujer y sus roles en el rubro. De este encuentro destacan conclusiones que buscan generar programas de capacitación vinculados a la formulación de proyectos, perfeccionar mecanismos de acceso a la información y nivelación de estudios, con el propósito de disminuir la brecha entre hombres y mujeres, y la segregación territorial. En 2008, el Taller Latinoamericano de Pesca Artesanalorganizado en Chile, concluye que el papel de las mujeres que se suman a las fases de captura, cultivos, transformación y comercialización pesquera, como es la representación político y sindical, convirtiendo este momento en un espacio de reflexión y encuentro respecto de "cómo el sector pesquero incrementa su contribución a la lucha contra la pobreza, la seguridad alimentaria, y el crecimiento económico, desde la perspectiva de las mujeres de la pesca artesanal incentivando la responsabilidad individual y colectiva de las familias y comunidades de pescadores y pescadoras artesanales para la construcción de su propio desarrollo" (CONAPACH, 2014). En esta oportunidad se consensuaron algunos roles de la mujer dentro del mundo pesquero artesanal, como la persistente lucha por espacios de participación en un mundo predominantemente masculino, convirtiéndose en un desafío para desarrollar modelos alternativos de liderazgo basados en la igualdad y complementación de géneros así como la expresión de capacidades diligénciales, actividades de negociación, diversificación productiva, valor agregado y comunicaciones, las que minimizarían los conflictos internos de poder. Mientras que los avances impulsados por el gobierno, a la fecha, han sido en torno de la capacitación y un registro estadístico con enfoque de género.

Estos nuevos roles de la mujer en la pesca artesanal, que se expanden hasta los espacios públicos de dominio político, pueden ser entendidos como respuestas territoriales en creciente desarrollo (Bennett, 2004). En este sentido, la participación de la mujer en la actividad sindical, ha comenzado a visibilizarse contemporáneamente, dejando atrás su caracterización como complemento del trabajo realizado por hombres.

Para el caso de la pesca y acuicultura la participación femenina tiene dos aristas, la primera es la sindicalización de mujeres que trabajan en plantas de proceso industriales, y aquellas mujeres sindicalizadas en 
agrupaciones independientes de pescadoras/es, algueras, encarnadoras, entre otras.

En el primer caso, la sindicalización de mujeres en plantas de proceso, SERNAPESCA (2011) reconoce el registro de 810 organizaciones inscritas en el R.P.A, de las cuales, 18 están compuestas exclusivamente por mujeres, 514 están compuestas por hombres y mujeres, 278 están constituidas exclusivamente por hombres y al menos 532 organizaciones cuentan con algún porcentaje de participación femenina. Las dos principales federaciones sindicales que congregan a trabajadores/as del salmón, FETRAINPES y CONATRASAL, declaran que cerca de 70\% de sus afiliados corresponden a mujeres. Dicha cifra es incluso mayor a la tasa de feminización general de la industria salmonera (60\% de acuerdo a Pinto y Kremerman, 2005). En el segundo caso, la participación femenina en organizaciones como sindicatos o en sistemas concesionados de áreas de manejo, entre 2010-2012, se reconocen 294 organizaciones, de las cuales 162 son mixtas y 130 están compuestas exclusivamente por hombres. Los porcentajes de participación en organizaciones sindicales y los roles dirigenciales femeninos no se condicen, tanto en sindicatos de pescadores/as artesanales, como en los asociados a la industria de la acuicultura. En esta línea, Ganga y Allendez (2007), señalan que para el año 2004 apenas el 32\% de los/as líderes sindicales fueron mujeres, sin embargo estas se concentran en cargos como tesoreras y secretarias, accediendo sólo al 20,37\% de la presidencia de sus sindicatos, lo que convierte a la dirigente de la Confederación Nacionales de Pescadores Artesanales de Chile (CONACH), Zoila Bustamante, como una excepción.

En el contexto de crisis de la pesca artesanal y la orientación de las políticas públicas que potencian el turismo de intereses especiales, la transformación de materias primas para aumentar el valor agregado, no planteamos la pérdida de valor cultural y económico, o la conversión productiva de los pescadores artesanales, sino que planteamos a un actor invisible como visible. La particular posición de las mujeres en sociedades pesqueras es difícil de eludir, debido a que su estrecho vínculo con actividades como marisqueo, transformación y conservación, estiba/desestiba, preparación de la embarcación, avituallamiento, comercialización de materia prima, reparación de redes y aparejos; además de las actividades agro-ganaderas realizadas como medio de auto-subsistencia (Broullón,2011).

El trabajo realizado por las mujeres en la frontera/interface de la actividad es considerado de menor valor y como complementario a la actividad verdaderamente importante que es la extracción dentro del mar. Es un actor privado y público, capaz de relacionar la cadena de valor de la actividad pesquera artesanal, superando obstáculos vividos cotidianamente, capaces de perpetuar formas sustentables de alimentación familiar a través de la transformación de los productos extraídos cotidianamente del mar. Este actor se convierte en reflejo de una opción de desarrollo, involucrando en ello la decisión consciente de poner el énfasis en la protección del medio ambiente, el despliegue de la solidaridad y la necesidad de "profundizar la 
democracia”, (Avendaño, 2010), al hacer hincapié en la promoción explicita de los derechos y garantías sociales, es decir, un Buen Vivir.

\section{Consideraciones finales}

La mujer en la pesca artesanal como un actor históricamente invisibilizado, ha desarrollado su quehacer desde una cotidianidad paralela a los grandes modelos de desarrollo, incorporándose en las últimas de manera importante debido a las tensiones y crisis mismas del modelo.

El aumento de participación formal de la mujer en la cadena productiva de la pesca artesanal, es reconocer las nuevas necesidades que se abren en este proceso de crisis. Al verse en riesgo la entrada de ingresos al núcleo familiar, las mujeres se han visto obligadas a cumplir, junto con los hombres, el rol de proveedoras, transformando de esta forma las dinámicas de género asociadas al trabajo. Es así, como la salida al espacio público, más que una reflexión sobre los roles de género impuestos, se presenta principalmente, en su inicio, como una salida de emergencia a la crisis, visibilizando la capacidad de agencia de estos nuevos actores, se conviertan en portadores de imaginarios que propongan alternativas al desarrollo diseñado hasta el momento y pensar en un Buen Vivir de los territorios costeros, vistos como sistemas culturales con la naturaleza, y no como un medio de producción en la naturaleza.

En las dos últimas décadas, algunas organizaciones e instituciones internacionales han hecho un creciente reconocimiento de la participación de la mujer en la pesca artesanal. Este reconocimiento reafirma la invisibilidad histórica que han vivido las diversas mujeres en estos territorios, no obstante, su caracterización ha sido documentada enregistros etnográficos, los que grafican las cotidianidades que las mujeres en la pesca artesanal han desarrollado a través de las transformaciones territoriales, convirtiéndose en potencialidadades que permiten asumir, desde un enfoque con pertinencia territorial y cultural, la sustentabilidad de la pesca artesanal.

En 2015, Chile promueve la creación del Ministerio de la Mujer y la Equidad de Género, el que debiera considerar a la mujer en la pesca artesanal como garante en la integración de forma transversal los derechos y roles ejercidos. No obstante, es indispensable sumar al enfoque tradicional de políticas públicas, el entender a la pesca artesanal como un sistema cultural, siendo compatible a la noción de Buen Vivir.

El Buen Vivir puede ser un aporte potencial en las Políticas Públicas, e implica una estrecha colaboración entre instituciones, ONG's y organizaciones territoriales. Implicaría avanzar en enfoques participativos, conciliadores entre conocimientos, en el auto reconocimiento y puesta en valor de mujeres organizadas en las diversas funciones que ejercen, como cultoras, pescadoras, recolectoras, dirigentes, dueñas de casa y muchas veces únicas proveedoras de sus familias para ser parte del futuro sustentable de la pesca artesanal. 


\section{Notas}

${ }^{1}$ Parte del Proyecto FONDECYT 1121204 “Geoantropología de los imaginarios del mar interior de Chiloé: itinerarios de temporalidades y apropiaciones socioculturales marítimas" y reflexiones en el IV CEPIAL, en la mesa redonda "La dirigencia de la mujer en la Pesca artesanal”. Enero de 2015, Osorno, Chile.

${ }^{2}$ El 100\% de los funcionarios administrativos de las salmoneras en Magallanes son hombre, mientras que en las plantas de proceso, el $80 \%$ de los trabajadores son mujeres (Chávez y Milihuichún, 2011: 97). 


\section{Bibliografía}

Abramo, L. (2004). ¿Inserción laboral de las mujeres en américa latina: una fuerza de trabajo secundaria?.Revista Estudos Feministas, 12(2), 224- 235.

Andrade, B., Arenas, F., y Guijó, R. (2008). Revisión crítica del marco institucional y legal chileno de ordenamiento territorial: el caso de la zona costera. Revista de Geografía Norte Grande, (41), 23-48

Arteche, S., y Saavedra, G. (2014). Transformaciones en la dimensión subjetiva del trabajo en mujeres laborantes de la industria Salmonera de Quellón y Puerto Cisnes, Sur-Austral de Chile. Trabajo presentado en Pre ALAS Argentina 2014, Buenos Aires, Argentina.

Avendaño, O. (2010). El buen vivir. Una vía para el desarrollo.Polis, Revista Latinoamericana, 9 (25),557-561.Recuperado dehttp:// polis.revues.org/260

Rojas, B. (2007). La mujer y el mar: su reto laboral en la costa oriental venezolana. En Y. Delgado y M.C. González (comp.) Mujeres en el mundo: historia, revoluciones, dictaduras, trabajo, religión y poesía, (pp.307- 320). Venezuela: Laboratorio de Investigaciones en Estudios del Trabajo (LAINET)

Bennett, E. (2005). Gender, fisheries and development. Marine Policy 29 (5), 451-459.

Breton, Y.y Doyon, S. (1999). La noción de "familia" en la antropología marítima: del parentesco al manejo costero. Revista Perspectivas rurales, (6), 37- 45. Recuperado de https://www.perspectivasrurales.org/ index.php/component/jdownloads/summary/13-perspectivas-6/106la-nocion-de-la-familia-en-la-antropologia

Broullón, E. (2011). La política sexual y la segregación ocupacional en las sociedades pesqueras.Revista Estudos Feministas, 19 (1), 73-89. Recuperado de http://www.redalyc.org/articulo.oa?id=38118774006

Chávez, E. \&Milihuichún, C. (2011). Los efectos socioeconómicos de la salmonicultura en la región de Magallanes. (tesisde pregrado).Universidad de Magallanes, Punta Arenas, Chile.

Cid,B. (2012). Maternizando lo político: mujeres y género en el movimiento sindical de la industria salmonera chilena. Revista Estudos Feministas, 20(1), 189-207. Recuperado de http://www.redalyc.org/ articulo.oa?id=38122296011

Colectivo Internacional de Apoyo a los Pescadores Artesanales. (2008). Derechos de género en la Pesca Artesanal de Latinoamérica. Reali- 
zado por Ecoceanos.cl, en base a información de FAO, de diversas organizaciones de la sociedad civil y de los encuentros de pescadores y pescadoras artesanales realizados en Chile, Taller Latinoamericano de la Pesca Artesanal, y en Tailandia, Conferencia Internacional de Pesca de Pequeña Escala

CONAPACH.(2014). Conclusiones del primer encuentro internacional de mujeres de la pesca artesanal y acuicultura de pequeña escala (2007)IFOP.Primer Congreso Internacional de Pescadoras Artesanales y Acuicultoras de Pequeña Escala, Valparaíso, Chile.

Cruz, J. y Argüello, F. (2006). Paradigmas de la antropología en el estudio de las sociedades costeras.Revista mad0 (15), 27-45. doi:10.5354/ 0718-0527.2006.14019

FAO.(2009). El estado mundial de la pesca y la acuicultura -2008.Recuperado dehttp://www.fao.org/docrep/011/i0250s/i0250s00.htm

Frangoudes, K \&Keromnes, E. (2008).Women in Artisanal Fisheries in Brittany, France. Development, 51 (2) 265-270. doi:10.1057/ dev.2008.20

García, A. (2001). Género en la pesca artesanal en el Perú. Estudios Antropológía.Revista Investigaciones Sociales, 5 (7). 43 - 63. Recuperado de http://sisbib.unmsm.edu.pe/BibVirtualdata/publicaciones/ inv_sociales/N07_2001/a03.pdf

Ganga, F y Allendez, E. (2007).Análisis teórico del sindicalismo en la Región de los Lagos. Universum,22 (2), 125-150. Recuperado de http:/ /universum.utalca.cl/contenido/index-07-2/art8.html

Gordon, E. (1991). La mujer costeña en la pesca artesanal. Wani Revista del Caribe Nicaragüense, 0 (9), 56 - 73. Recuperado de http:// revistas.bicu.edu.ni/index.php/wani/article/view/281/278

Guerra, D.,Skewes, J.C., Pino, D. y Pilquiman, M.(2010). Discursos y soberanía local: Pensadoras y pensadores de la cordillera de la costa del sur de Chile frente a la expansión capitalista. Aljaba, 0 (14)31-49. Recuperado de http://www.scielo.org.ar/pdf/aljaba/v14/v14a02.pdf

Harper, S., Zeller, D.,Hauzer, M.,Pauly, D\&Sumaila, U. (2013). Women and fisheries: Contribution to food security and local economies. Marine Polic39, 56-63.

Hidalgo, C., Ther, F. y Saavedra, G. (2013). Oscilaciones en la temperatura de prácticas pesquero-artesanales: la expansión de la economía de capitales en las Caletas de Islas Huichas, Puerto Cisnes y Puerto Melinka, Región de Aysén; Chile. Revista Sémata Ciencias Sociais e Humanidades, (25), 223-242. Recuperado de http:// 
Hirata, H y Kergoat, D. (2007). Novas configurações da divisão sexual do trabalho. Cadernos de Pesquisa, 537 (132), 595-609. Recuperado de http://scielo.br/pdf/cp/v37n132/a0537132

López, L. (2011). Estudio de caso sobre el rol de las mujeres en comunidades pesqueras del Golfo de Fonseca, Marcovia, Choluteca (tesis de pregrado) Universidad de Zamorano,Tegucigalpa, Honduras.

Maneschy, M., Siquira, D., Miranda, M. (2012). Pescadoras: subordinação de escadoras: subordinação de gênero e empoderamento gênero e empoderamento. Revista Estudos Feministas, 20(3), 713-737. Recuperado de http://www.redalyc.org/articulo.oa?id=38124755007

McPhee, B. (2013). ¿Cuán nuevas son las ruralidades chilotas? Transformaciones territoriales en Chiloé central, 1980 - 2012 (tesis de maestría). Pontificia Universidad Católica de Chile, Santiago, Chile.

Pilquiman, M. y Skewes, J.C. (2010). Relatos en torno al etnoturismo: La comunidad indígena de Choroy-Traiguén, Provincia de Osorno, y sus proyectos de desarrollo. Revista Lider, 12 (16), 105-124. Recuperado de http://ceder.ulagos.cl/lider/images/numeros/16/ [LIDERVol16A\%C3\%B1o12-2010-ISSN-0717-0165]7.-Relatosen tornoaletnoturismo.pdf

Pilquiman, M. y Skewes, J.C. (2009). Los paisajes locales y las encrucijadas del etnoturismo: reflexiones a partir de los proyectos turísticos de comunidades indígenas de la región de Los Lagos en Chile. Cuadernos de Turismo, 0 (24), 169-191. Recuperado de revistas.um.es/ turismo/article/download/92701/89181

Pinto, F., y Kremerman, M. (2005). Cultivando pobreza: condiciones laborales en la salmonicultura. Publicaciones Terram. $R P P N^{\circ} 18$. Recuperado de http://www.terram.cl/images/RPP/ rpp18_cultivandopobreza.pdf

Ram-Bidesi, V. (2015). Recognizing the role of women in supporting marine stewardship in the Pacific Islands. Marine Policy 59 (5), 1-8

Rebolledo, L. (2012). Resistencia y cambios identitarios en trabajadores/as del salmón en Quellón. Polis, Revista Latinoamericana, 11 (31), 223239. Recuperado de http://polis.revues.org/3714

Rodríguez, E. (2011). La pesca artesanal como mundo de vida desde una perspectiva de género. Comunidad de punta colorada. Municipio cruz salmerón acosta-estado Sucre. (Tesis de pregrado). Universidad de Oriente, Sucre, Venezuela. 
Saavedra, G. (2015). Los futuros imaginados de la pesca artesanal y la expansión de la salmonicultura en el sur austral de Chile. Chungará Revista de Antropología Chilena, 47 (3), 00-00. Recuperado de http:/ /www.scielo.cl/pdf/chungara/v47n3/aop3115.pdf

Rubio-Ardanaz, J. (2005).“Sardineras: visión antropológica económica de una forma de distribución del pescado”. LAN HARREMANAK, Revista de relaciones Laborales. Número 13. Páginas 177-198

Servicio Nacional de Pesca y Acuicultura.(2014). Indicadores de estadísticas pesqueras con enfoque de género a nivel sectorial. Recuperado de http://www.sernapesca.cl/

Servicio Nacional de Pesca y Acuicultura. (2011)Mujeres y hombres en el sector pesquero y acuicultor de Chile. Recuperado de http:// www.sernapesca.cl/

Subsecretaría de Pesca y Acuicultural, 2015. Decreto Exento N² 257-2015 Rectifica D EX No 39-2015 Establece Porcentaje de desembarque de especies como Fauna acompañante de Recursos que indica, año 2015. (Publicado en Diario Oficial 16-04-2015)

Torres, M.P y Saavedra, G. (2014). Las salmoneras y la economía del hogar pesquero artesanal en el mar interior de Chiloé, Chile: Las subjetividades del lugar en tiempos del desarrollo. Trabajo presentado en Pre ALAS Argentina 2014, Buenos Aires, Argentina.

Woortmann, E. (2007). Cambios de tiempo y espacio/ cambios sociales, bajo el impacto de la modernización. Revista Estudos Feministas, 15 (2) 476-484. Recuperado de http://www.redalyc.org/ articulo.oa?id=38115215

Yánez, R. (2010). Transformaciones identitarias en el archipiélago de Chiloé. Una discusión en torno al concepto identidad y su relación con los procesos de cambio (tesis de pregrado). Universidad de Chile, Santiago, Chile. 